\title{
5 GEFLÜCHTETE KINDER UND JUGENDLICHE IN DER Pflichtschule
}

Die Integration geflüchteter Kinder und Jugendlicher in die Pflichtschule stellte im deutschsprachigen Raum lange Zeit ein Randthema innerhalb sozialwissenschaftlicher Forschungen dar. Noch im Jahr 2009 konstatierten beispielsweise BEHRENSEN und WestPhaL, dass junge Geflüchtete in der deutschen Migrations- und Bildungsforschung ein „blinder Fleck“ sind. Die Autorinnen betrachteten dies als Ergebnis eines mangelnden politischen Willens sie als Bildungssubjekte anzuerkennen. Sie wiesen überdies darauf hin, dass die damit zusammenhängende geringe Forschungsförderung das Forschungsfeld für Wissenschaftler/innen nicht besonders attraktiv mache (BEHRENSEN \& WestPhal 2009, p. 46). Inzwischen hat Europa eine mit früheren Dekaden kaum vergleichbare Zuwanderung von Geflüchteten erlebt. Darunter befinden sich auch viele Kinder und Jugendliche, die nun eine Pflichtschule besuchen. Wenngleich die Fluchtbewegungen seit 2015 eine Zäsur darstellen und der Bedarf an sozialwissenschaftlicher Fluchtforschung (unter anderem im Bildungsbereich) deutlich sichtbarer geworden ist, bleibt die Anzahl der Studien zu diesem Thema nach wie vor überschaubar. Vor allem für den nationalen Kontext Österreich existieren kaum wissenschaftliche Publikationen, die sich explizit mit der Integration geflüchteter Kinder und Jugendlicher in der Pflichtschule beschäftigen. Einen knappen Überblick über die rezenten Zusammenhänge und Problemfelder bei der Beschulung geflüchteter Kinder und Jugendlicher in Österreich liefert Langthaler (2016). Für Deutschland vermittelt der Sammelband „Ankommen in der Schule: Chancen und Herausforderungen bei der Integration von Kindern und Jugendlichen mit Fluchterfahrung“" (McELVANY et al. 2017) einen übersichtlichen Abriss der Thematik. Benholz, Frank und Niederhaus (2016) sowie Preuss (2018) widmen sich ebenfalls der Integration marginalisierter Schüler/innengruppen in Deutschland, wobei geflüchtete Kinder und Jugendliche eine Teilgruppe ihrer Analyse darstellen. LÜTJE-KLOSE et al. (2017) thematisieren den gesteigerten Bedarf an der Entwicklung inklusiver Strukturen in den Schulsystemen Deutschlands, Österreichs und der Schweiz. Aufgrund der erheblichen Ähnlichkeiten der nationalen Schulsysteme in Deutschland und Österreich ${ }^{13}$ werden einige für Deutschland getroffene Analysen auch für die empirischen Beispiele aus Österreich verwendet.

\footnotetext{
13 Sowohl Österreich als auch Deutschland differenzieren sehr früh, bereits nach vier Jahren eines gemeinsamen Unterrichts, in weiterführende Schultypen aus. In beiden Staaten ist Deutsch die Unterrichtssprache und beide weisen ähnliche Entwicklungen im Bereich Zuwanderung auf (angefangen mit der sogenannten „Gastarbeitermigration“ bis hin zu den rezenten Fluchtmigrationsbewegungen nach Europa).
} 


\section{1 „Passungsprobleme“ bei der Beschulung nichtdeutschsprachiger Schüler/innen}

Der „monolinguale Habitus“, die frühe Ausdifferenzierung des österreichischen Bildungssystems auf struktureller Ebene sowie der sozioökonomische Status der Eltern haben maßgeblichen Einfluss auf die Bildungschancen der Schüler/innen (vgl. Kapitel 3). In vielerlei Hinsicht gelingt es nicht, einen positiven Umgang mit Diversität beziehungsweise ungleichen Startvoraussetzungen zu finden. Insbesondere im Hinblick auf sprachliche Vielfalt ist eine Defizitorientierung bestimmend. Mehrsprachigkeit (vor allem Kenntnisse „,nichtwestlicher Sprachen“ betreffend) findet innerhalb der Strukturen des derzeitigen Bildungssystems kaum Anerkennung. Pilz (2016, p. 59) weist darauf hin, dass Mehrsprachigkeit als individuelles Problem betrachtet wird und nicht im Curriculum verankert ist.

PILz (2016), die sich mit der Benachteiligung nichtdeutschsprachiger Schüler/ innen im deutschen Schulsystem auseinandersetzt, verwendet einen interessanten holistischen Ansatz, um die Chancenungleichheit dieser Kinder und Jugendlichen zu erklären. Sie identifiziert sogenannte „Passungsprobleme“ auf der Makro-, Mesound Mikroebene. Zur Makroebene zählen die Strukturen des Bildungssystems, die in Kapitel 3 bereits ausführlich dargelegt worden sind. Kinder und Jugendliche mit nicht ausreichenden Deutschkenntnissen ,passen“ nicht in das Schulsystem, weil Deutsch die Voraussetzung für erfolgreiche Bildungsteilhabe darstellt und frühe Selektionsmechanismen mit dem Ziel leistungshomogene Gruppen zu bilden dazu beitragen, dass dieses „Defizit“ nicht zeitgerecht ausgeglichen werden kann. ${ }^{14}$

Dabei soll nicht der Eindruck erweckt werden, dass neu zugewanderte Kinder und Jugendliche nicht die Landessprache erlernen sollten oder Deutsch als Unterrichtssprache im Einwanderungsland Österreich keine Berechtigung mehr hätte. Jedoch weisen Wissenschafter/innen darauf hin, dass eine Nichtanerkennung der Kompetenzen in ihrer Erstsprache weitreichende Konsequenzen auf das Selbstkonzept der Schüler/ innen haben kann, was sich wiederum allgemein negativ auf die Lern- und Leistungsfähigkeit auswirken könnte (PILz 2016, p. 81). Erfolge in der Schule sind nämlich mit Ausnahme von nicht sprachbasierten Fächern wie Sport oder Bildnerische Erziehung kaum möglich. Neu zugewanderte Kinder könnten somit zu der Selbsteinschätzung gelangen, dass sie über keine nennenswerten Kompetenzen verfügen, weil sie die deutsche Sprache noch nicht ausreichend beherrschen.

Die strukturellen Gegebenheiten sind für sich genommen jedoch noch nicht allein aussagekräftig. Entscheidend ist des Weiteren, wie mit Mehrsprachigkeit am jeweiligen Schulstandort von der Schulleitung und dem Kollegium (Mesoebene) umgegangen wird (PILz 2016, p. 64). Umgangssprachlich könnte man dies damit ausdrücken, wie

14 Hierbei könnte ein gesteigertes Angebot an Ganztagsschulen für Kinder mit nichtdeutscher Erstsprache ein vielversprechender Lösungsansatz sein (vgl. PILz 2016, pp. 52 f.). Hinsichtlich weiterer Merkmale auf der Makroebene vgl. ebd. pp. 48 ff. 
aufgeschlossen eine Schule für Diversität ist beziehungsweise welches „Klima“ diesbezüglich besteht. Jede Schule ist durch institutionalisierte Routinen, Gewohnheiten und interne Logiken charakterisiert. Wenn an einem Standort kein konstruktiver Umgang mit Heterogenität gelingt, spricht PILZ von ,institutioneller Diskriminierung“ (ebd.).

Schließlich wird auf der Mikroebene der Umgang mit Diversität von Lehrer/ inne/n, Schüler/inne/n sowie deren Umfeld analysiert. Einerseits geht es um die individuelle interkulturelle Kompetenz der Pädagog/inn/en. Dieser Aspekt kommt PILz (2016, pp. 72, 76) zufolge in der Lehrer/innen/aus- und -fortbildung zu kurz. LUCIAK und BINDER (2010) machen ebenfalls darauf aufmerksam, dass interkulturelle Pädagogik institutionell noch wenig verankert ist. Sie liefern mit ihrem „Handbuch Interkulturelles Lernen“" praxisnahe Beispiele und didaktische Ansätze zur Umsetzung im Unterricht. Andererseits werden kulturelle Differenzen der Schüler/innen und sich daraus ergebende Probleme als individuelle Passungsprobleme zwischen der Sozialisation der Kinder und den Erwartungshaltungen der Schule interpretiert. Der Grund schulischen Versagens wird bei den Kindern und Jugendlichen sowie in deren familiärem Umfeld gesucht, nicht in der Schule als Organisation, beim Unterricht oder den Lehrer/inne/n.

„Da die Schule einen passenden, heißt schulische Aufgaben unterstützenden, familialen Kontext voraussetzt, wird Ungleichheit geschaffen, da diejenigen, die die Normalitätserwartungen erfüllen, im Bildungssystem erfolgreicher sind." (PILZ 2016, p. 82)

Wie diese drei Ebenen ineinandergreifen, zeigt sich sehr gut an den empirischen Beispielen, die in den folgenden Abschnitten dargelegt werden. Obwohl das Schulsystem und die Bildungsverwaltung die Rahmenbedingungen vorgeben, besitzen Schulleiter/innen und Lehrer/innen an den jeweiligen Standorten dennoch Handlungsmacht und haben einen gewissen Gestaltungsspielraum, wenn es um die Integration nichtdeutschsprachiger beziehungsweise geflüchteter Schüler/innen geht. Außerdem müssen sie oftmals kreative und unbürokratische Wege finden, da sie vom Dienstgeber nur wenig Unterstützung erhalten. Dies verlangt oftmals viel Eigenengagement und Ad-hoc-Lösungen. Obwohl die folgende Analyse auf die strukturellen Rahmenbedingungen des (Miss-)Erfolgs von Schüler/inne/n fokussiert, soll an dieser Stelle nicht unerwähnt bleiben, dass dieser auch mit individuellen (sprachlichen) Begabungen sowie der persönlichen Motivation zusammenhängt, was jedoch im Rahmen dieser Forschung nicht erhoben werden konnte.

\subsection{Herausforderungen und Bewältigungsstrategien in der Pflichtschule}

In den beiden beforschten Regionen in Niederösterreich wurden insgesamt drei Schulleiter/innen von Neuen Mittelschulen und zwei Volksschuldirektorinnen interviewt. Zusätzlich wurde ein Interview mit einer DaF/DaZ-Förderlehrerin der Deutsch- 
förderkurse und ein Gruppeninterview mit drei ehrenamtlichen Senior/inn/en-Lehrer/ inne/n geführt.

Beide Regionen, sowohl die drei Gemeinden im Mostviertel als auch St. AndräWördern, hatten zum Erhebungszeitpunkt im Jahr 2018 nur vereinzelt geflüchtete Kinder aufgenommen. Ganze Klassen mit einer beträchtlichen Anzahl an geflüchteten Schüler/inne/n beziehungsweise mit Kindern und Jugendlichen mit nichtdeutscher Erstsprache, wie dies aus urbanen Ballungszentren bekannt ist, gab es dort nicht. Diese Tatsache wurde weitgehend als positiv betrachtet, barg aber auch einige Herausforderungen. Hierfür waren eigene Konzepte für die Integration dieser Schüler/innen gefragt, da zusätzliche Ressourcen vom Dienstgeber nicht vorgesehen waren oder eingefordert werden mussten. Wie mit dieser Situation an den einzelnen Schulstandorten umgegangen wurde, wird in den folgenden Abschnitten dargelegt.

\subsubsection{Das Problem der altersgemäßen Einstufung}

Schulpflichtige Kinder sind in Österreich altersgemäß einzustufen, wobei plus/ minus ein Jahr auf- oder abgestuft werden darf. Sofern keine ausreichenden Deutschkenntnisse vor-gewiesen werden können, dürfen Schüler/innen maximal zwei Jahre als außerordentlich geführt werden, wobei sie kein Zeugnis, sondern eine Schulbesuchsbestätigung erhalten (SchUG § 4 Abs. 3). Das Abschlusszeugnis der neunten Schulstufe ist insofern zentral, als es die Voraussetzung für weitere Ausbildungsmöglichkeiten, etwa eine Lehre, darstellt. Kommen die Geflüchteten im noch schulpflichtigen Alter, zum Beispiel mit 14 Jahren, nach Österreich, dann müssten sie innerhalb von zwei Jahren zu ordentlichen Schüler/innen werden, damit sie benotet werden und ein Abschlusszeugnis erhalten können. Besonders bei bereits älteren Schüler/inne/n stellt die Integration in den Regelunterricht jedoch eine beträchtliche Herausforderung dar, da sich unzureichende Sprachkenntnisse nicht nur im Fach Deutsch, sondern auch in den meisten anderen Fächern als hinderlich bei der Aneignung fachlicher Inhalte erweisen. Engagierte Direktor/inn/en versuchen allerdings Wege zu finden, um den Jugendlichen „nicht die Zukunft zu verbauen“, was manchmal nicht gesetzeskonforme Entscheidungen verlangt (Interview DiREKTOR NMS GEMEINDE B 2018).

„, Aktuell habe ich einen Schüler-nach zwei Jahren sollte man ordentlicher Schüler werden - der Schüler war aus Afghanistan. War zuerst [...] 20 Monate in [einer anderen Gemeinde], hat dort im Unterricht nicht recht folgen wollen. Und als er zu mir kam vor eineinhalb Jahren, sollte ich ihn eigentlich schon zum ordentlichen Schüler machen, aber hätte ich den gemacht, hätte er beurteilt werden müssen und er hätte eigentlich außer Turnen und Werken, Musik [...], hätte er wahrscheinlich nur Fünfer gehabt. Der ist jetzt noch außerordentlicher Schüler, was nicht gesetzeskonform ist. " (Interview DireKTOR NMS GemEINDE B 2018)

Schulleiter/innen sind bei der Aufnahme von asylwerbenden Kindern immer wieder damit konfrontiert, dass diese während des Schuljahres eingestuft werden 
müssen oder aber die Schule plötzlich wechseln (vgl. HeINTZE 2017, p. 187). Besonders in ländlichen Gemeinden ist ein positiver Asylbescheid ein wesentlicher Grund dafür, die Gemeinde zu verlassen, was auch einen Schulwechsel bedeutet. Doch auch eine drohende Abschiebung oder ein Umzug aufgrund der Verlegung in ein anderes Quartier können zu einem spontanen Ortswechsel führen. Die Direktor/inn/en sowie die Pädagog/ inn/en stehen vor der Herausforderung, diese Kinder und Jugendlichen sowohl sozial zu integrieren als auch im Hinblick auf den Bildungsauftrag bestmöglich zu fördern. Selbst wenn allen geflüchteten Kindern und Jugendlichen in der Regel gemeinsam ist, dass sie ohne Deutschkenntnisse eingeschult werden, weisen sie dennoch in anderen bildungsbiografischen Bereichen eine enorme Heterogenität auf (von Dewitz \& Massumi 2017, pp. 29 f.; PreUss 2018, pp. 27 f.).

\subsubsection{Mangelnde Ressourcen als Herausforderung}

Für ländliche Schulstandorte mit wenigen neu zugewanderten Kindern gilt häufig, dass sie im Vergleich zu Schulen mit konstant hohen Zahlen an außerordentlichen Schüler/inne/n bei der Vergabe von zusätzlichen Ressourcen durch den Landesschulrat nicht berücksichtigt werden. In der Praxis stellen jedoch schon einzelne Kinder, die zusätzliche Förderung benötigen, die Schulleitung vor die Frage nach der Bewerkstelligung dieser Aufgabe. Vor dem Hintergrund der empirischen Ergebnisse ist die Sinnhaftigkeit einer Problemdefinition, die auf Quantität abstellt und daraus ihre Handlungslogik legitimiert, kritisch zu hinterfragen.

In den Interviews wurde deutlich, dass sowohl die Volkschulen als auch die Neuen Mittelschulen auf „externe Ressourcen“, also ehrenamtliche Unterstützung, zurückgreifen mussten, da diese von staatlicher Seite nicht bereitgestellt wurden. In diesem Zusammenhang wurde auch das starre, bürokratische Schulsystem thematisiert, wobei der Landesschulrat beispielsweise eine Jahresplanung für die Ressourcenverteilung verlangt. Angesichts der bereits angesprochenen Situation, dass Schüler/innen zu unvorhersehbaren Zeiten aufgenommen werden müssen, ergeben sich Schwierigkeiten bei der Genehmigung.

„,Den Förderunterricht haben eigentlich nur die eigenen Leute gemacht. [...] Das hat [...] der Schule auf alle Fälle viel gebracht und ist eine Notlösung, weil der Staat selber oder das Bundesland selber nicht in der Lage ist, etwas Gescheites zu machen. [...] Sie verstecken sich hinter dem Argument, Ich brauche eine Planung für das ganze Jahr' und wenn ich das nicht sicherstellen kann, dass ich immer gleich viele Schüler habe und so und so unterrichte, dann gibt es halt nichts. "“ (Interview DireKTOR NMS GEMEINDE B 2018)

Im Rahmen der Schulbuchaktion, einer familien- und bildungspolitischen Leistung, die vom Bundesministerium für Arbeit, Familie und Jugend (BMAFJ) und dem Bundesministerium für Bildung, Wissenschaft und Forschung (BMBWF) getragen wird, werden Schüler/innen jährlich kostenlos mit Lehrbüchern ausgestattet. Hierfür steht den Schulen pro Schüler/in ein Budget zur Verfügung (BUNDESMINISTERIUM FÜR 
Arbeit, Familie und Jugend \& Bundesministerium für Bildung, Wissenschaft UND Forschung o. J.). Auch für außerordentliche Schüler/innen können Materialien bestellt werden, allerdings ist dies für die Schulen schwieriger zu planen, da die Kinder und Jugendlichen eher spontan aufgenommen werden müssen. Für den Direktor der NMS in der Gemeinde B bedeutete dies, dass er sich bei Bedarf direkt beim Verlag meldete und um passende Materialien bat. Eine solche Vorgangsweise ist in der Regel auch kein Problem, aber das Bestellsystem selbst ist auf diese speziellen Bedürfnisse der Schulen nicht ausgerichtet.

„Ja, also [...] eigentlich, wenn man fragt, gibt es nirgendwo verschlossene Türen. Aber das Schulsystem selber, die Verwaltung, stoßt halt an ihre Grenzen, weil sie so starr ist. Und man braucht - bevor ein Schüler beginnt-, braucht man alles schon fertig. Und jedes Kind ist eine Nummer und jedes Kind bekommt das und das zugewiesen. Und da darf im Schuljahr drinnen nichts mehr passieren. “ (Interview DiREKTOR NMS GEMEINDE B 2018)

Das strategische Vorgehen ersetzt allerdings kein flexibleres System, sondern ist als Ad-hoc-Lösung von Seiten einzelner Schulstandorte zu sehen. Im Idealfall stellen Bildungs-verwaltung und der einzelne Schulstandort nämlich eine Verantwortungsgemeinschaft dar. Dies wurde in jüngster Zeit insbesondere durch die Herausforderung der Integration von geflüchteten Kindern deutlich (Preuss 2018, pp. 46 f.).

Der Direktor der NMS in Gemeinde A zeigte zwar Verständnis für die Entscheidungen des Landesschulrats für eine äußerst geringe Anzahl von geflüchteten Kindern an einem Schulstandort keine weitere Lehrperson einsetzen zu können, betonte aber auch, dass man die Aufgabe der Zusatzförderung nicht ausschließlich den Ehrenamtlichen überlassen könne. An seinem Schulstandort fand die Zusatzförderung überwiegend durch Pädagog/inn/en statt, die in ihren Freistunden einzelne Schüler/innen aus dem Regelunterricht herausnahmen und mit ihnen Deutsch lernten. Er würde sich deshalb eine Art „Belohnungssystem“ wünschen, durch welches die ehrenamtlich geleistete Arbeit in irgendeiner Form honoriert werden würde (vgl. Interview DiREKTOR NMS Gemeinde A 2018).

HeINTZE (2017, p. 188) weist darauf hin, dass die Integrationsaufgabe von Schulen nicht ausschließlich im sprachlichen Bereich liegt, sondern auch auf gesellschaftliche Teilhabe abzielen sollte. Dabei geht es darum, Kindern und Eltern zu vermitteln, dass die Schule ein sicherer, diskriminierungsfreier Ort ist. Allerdings belegt ein Beispiel aus der Praxis, dass das Einsparen von Ressourcen nicht nur den Bereich Deutschförderung betrifft, sondern sich darüber hinaus auf Aktivitäten bezieht, die eigentlich die soziale Integration fördern sollen. Der Schulleiter der NMS in Gemeinde A schilderte ein Beispiel, wonach die Mutter eines geflüchteten Schülers ihren Sohn gerne am Schikurs teilnehmen lassen wollte. Aufgrund seiner geringen Deutschkenntnisse suchte die Schulleitung beim Dienstgeber um die Mittel für eine zusätzliche Begleitperson an, was jedoch abgelehnt wurde. Der Direktor entschied daraufhin, den Schüler nicht mitzunehmen: 
„Also wenn der dort irgendwo im Schigebiet verloren geht... Das ist eine Mörderverantwortung, die kann man keinem aufhalsen von den verantwortlichen Lehrpersonen. Weil wenn dann nämlich etwas passiert, dann heißt es: ,Wo waren die Lehrer und warum hat man da nicht Vorsorge getroffen?' Aber wenn man Vorsorge treffen will, dann wird es mitunter aus Kostengründen nicht genehmigt. So ist es halt. [...] Schule soll alles leisten, aber es soll nichts kosten. " (Interview DireKTOR NMS GEMEINDE A 2018)

In ähnlicher Weise schilderte die Schulleiterin der NMS in St. Andrä-Wördern das Dilemma mit Projektwochen und Wandertagen. Sie hatte die Erfahrung gemacht, dass die Eltern der geflüchteten Schüler/innen ihre Kinder an solchen Exkursionen ohnehin nicht teilnehmen lassen - insbesondere die Mädchen. Einerseits bedauerte sie dies, andererseits hatte sie auch Bedenken ein Kind auf Wanderungen und dergleichen mitzunehmen, welches kaum Deutsch versteht.

„Die fahren nicht mit, ist kein Thema. Und jetzt sag ich einmal so: Solange sie nicht sehr gut Deutsch können, ist es auch besser, wenn sie nicht mitfahren, weil, wir die Kommunikation nicht haben. Wenn ich mit dem Kind [...] eine Wanderung in eine Schlucht mache [...], dann muss ich mich darauf verlassen können, dass ich mit dem wirklich reden kann, wenn irgendetwas ist [...]. Oder wenn der in der Nacht aufwacht und schreit, ja? Ist das ein Trauma, tut dem was weh, was hat der oder die? Das muss ich wissen. " (Interview DireKTORIN NMS ST. ANDRÄWÖRDERN 2018)

Die Zitate zeigen, dass sich Lehrer/innen dieser zusätzlichen Verantwortung aufgrund von Verständigungsschwierigkeiten oft nicht gewachsen fühlen. Mitunter ist aber auch die Finanzierung der Projektwoche für ein/e geflüchtete/n Schüler/in ein Thema. Aus einer pragmatischen Sicht sind dies wichtige Faktoren, weshalb sich Schulleiter/innen nicht explizit für die Teilnahme an Projektwochen einsetzen.

Es gibt aber auch Schulleitungen, die sich außerordentlich darum bemühen, Möglichkeiten zur Teilhabe an sozialen schulischen Aktivitäten (zum Beispiel Projektoder Wandertage) für alle Kinder zu schaffen - unabhängig von der finanziellen Situation der Eltern. Im Mostviertel sponsern jeweils lokal ansässige Firmen die Schulen für diese Zwecke (Interview DireKTor NMS GEMEINDE A 2018; Interview DireKTOR NMS Gemeinde B 2018). Hier waren Engagement, Planung und guter Wille von Seiten der Direktion gefragt, um zum Beispiel die Teilnahme am Schikurs zu ermöglichen. Preuss (2018, p. 50) betont, dass Schulleitungen der Motor für eine inklusive Schulentwicklung sind. Dabei ist es wichtig sowohl schulische (Lehrkräfte, Schüler/innen, Eltern) als auch schulexterne Akteur/inn/e/n (zum Beispiel außerschulische Partner/innen, Gemeinden) in die Umsetzung der Vision einer inklusiven Schulkultur miteinzubeziehen, „die sich nicht nur an bildungspolitischen Aufträgen orientiert, sondern an einer langfristigen pädagogischen Vision“ (Preuss 2018, p. 48).

„,Und bei Sportwochen und Schikurs fahren alle mit. [...] Denn wenn alle österreichischen Kinder mitfahren, denen geholfen wird, sollte der auch mitfahren, weil, 
das ist eine besondere Lernwoche zur Integration. [...] Weil, der hat keine Ski und keinen Anorak. Natürlich tun wir da ein halbes Jahr vorher schauen, dass die Caritas - die hat ja zweimal im Jahr Bekleidungsaktionen -, dass er Fäustlinge, Schischuhe und Anorak, alles kriegt. Skifahren hat er zwar keinen Kurs noch gemacht, aber wir haben an der Schule eine Langlaufausrüstung. Und da nimmt er halt bei der Langlaufgruppe teil. " (Interview DIREKTOR NMS GEMEINDE B 2018)

Wenn Integration an Schulstandorten gut funktioniert, ist das in vielen Fällen dem guten Willen der Schulleitung und des Kollegiums zu verdanken. In Gemeinde B sind zum Beispiel lokal ansässige Unternehmen, die Diözese oder Verlage Kooperationspartner, die der Schulleiter der NMS in der Vergangenheit bei Bedarf um Unterstützung bat.

„,Wenn man ein bisschen ein Netzwerk hat, fragst du dort nach, da nach [...]. An Geld wird es eigentlich nie scheitern, aber an dem System. Dass eine Systematisierung da drinnen ist, an dem hat es schon gescheitert. Es ist so vieles immer abhängig vor Ort von den lokalen Initiativen und wie weit man bereit ist, selber zu gehen. " (Interview DireKTOR NMS GEMEINDE B 2018)

Diese Beispiele machen deutlich, dass auf struktureller Ebene Unterstützung für eine umfassende und tiefgreifende Teilhabe nicht vorgesehen ist. Das Schulsystem reagiert nur träge auf die äußeren Veränderungen. Die fehlende Flexibilität im System wird oftmals durch Einzelpersonen mit ihrem Engagement an den Schulstandorten ausgeglichen. In ähnlicher Weise ist dies bei der lokalen Integration von Geflüchteten in den Gemeinden zu beobachten. Ehrenamtliche leisten hierbei oftmals einen wesentlichen Beitrag (vgl. Kapitel 7).

\subsubsection{Möglichkeiten der Organisation zusätzlicher Ressourcen}

Von Dewitz und Massumi (2017, p. 32) unterscheiden fünf schulorganisatorische Modelle bei der Beschulung von geflüchteten Kindern und Jugendlichen: das submersive, integrative, teilintegrative und parallele Modell sowie das parallele Modell mit Schulabschluss. Während beim submersiven Modell die Sprachförderung im Regelunterricht stattfindet, schließen Schüler/innen die Schule im parallelen Modell mit Schulabschluss in einer speziell dafür eingerichteten Klasse ab. Bei den Mischformen kommt es auf die Dauer und Häufigkeit des separaten Unterrichts an. Beim integrativen Modell beschränkt sich dieser auf die Sprachförderung; die Schüler/innen nehmen grundsätzlich am Regelunterricht teil. Beim teilintegrativen Modell findet die spezifische Sprachförderung in einer eigens dafür eingerichteten Klasse statt, wobei die Kinder und Jugendlichen ansonsten im Rahmen des Regelunterrichts unterrichtet werden. Das parallele Modell sieht eine vorübergehende, zeitlich begrenzte separate Beschulung vor (vgl. Gruber 2017).

Ab dem Schuljahr 2018/19 wurden in Österreich Deutschförderklassen beziehungsweise -kurse eingeführt (vgl. BUNDESMINISTERIUM FÜR BILDUNG, WisSENSCHAFT 
UND ForsChung 2018). Die Deutschförderklassen entsprechen der Klassifikation nach dem teilintegrativen Modell, die Deutschförderkurse dem integrativen Modell. Allerdings sind diese Maßnahmen umstritten. Schulleiter/innen sind häufig geteilter Meinung, ob die optimale Förderung ihrer Schüler/innen außerhalb des Regelunterrichts sinnvoll ist oder nicht. Die Volksschuldirektorin in St. Andrä-Wördern war beispielsweise davon überzeugt, dass Kinder die Sprache am schnellsten lernen, wenn sie sozial gut integriert sind. Das Herausnehmen aus dem Klassenverband würde diesem Ziel daher nicht wirklich dienen.

Dass das Alter der Schüler/innen hinsichtlich der Form der Sprachförderung in Deutsch eine wesentliche Rolle spielt, wird auch durch wissenschaftliche Forschung untermauert. Je älter neu zugewanderte Schüler/innen zum Zeitpunkt ihrer Einschulung in Österreich sind, desto effektiver dürften differenzierte Sprachfördermodelle sein, die gezielte, separate Förderung vorsehen (vgl. GRUBER 2017; RoTHWEILER \& RUBERG 2011, pp. 10 f.). Es wird davon ausgegangen, dass man ab etwa einem Alter von zehn Jahren beim Lernen einer Zweitsprache vom „Zweitsprachenerwerb Erwachsener“ sprechen kann, der anders funktioniert als bei jüngeren Kindern ${ }^{15}$, die schon sehr früh mit einer Zweitsprache in Berührung kommen (RothweILER \& RUBERG 2011, pp. 8 f.). Dementsprechend werden vor allem NMS vor die Frage gestellt, wie der Zweitsprachenerwerb am besten gefördert werden soll. In höheren Schulstufen fällt es den Schüler/inne/n mit sehr geringen Deutschkenntnissen deutlich schwerer, den vermittelten Inhalten in den anderen Fächern zu folgen. Die Beherrschung der Sprache ist nämlich die Voraussetzung zur Aneignung von Fachwissen (GRUBer 2018, p. 17). Hierbei scheint ein submersiver oder integrativer Ansatz nur schwer umsetzbar und wird meist als nicht sinnvoll erachtet. In diesem Fall könnte durch den Besuch einer separaten Deutschklasse (teilintegratives Modell) eine sprachliche Basis gelegt werden. Dies mit dem Ziel, möglichst schnell am Regelunterricht partizipieren zu können. Allerdings braucht es für die Genehmigung der Deutschförderklassen und -kurse eine Mindestanzahl an förderbedürftigen Kindern und Jugendlichen, die viele ländlich gelegene Schulstandorte mit stets wenigen außerordentlichen Schüler/inne/n nicht erreichen.

Bei jüngeren Kindern im Volksschulalter bestehen noch nicht so ausgeprägte Differenzen, was den Wortschatz und das Fachwissen betrifft. Daher können in Volksschulen mit einem spielerischen Ansatz rasche Fortschritte in der Sprache erzielt werden. An der Volksschule in St. Andrä-Wördern wird auch Nachmittagsbetreuung angeboten. Da Kinder maßgeblich durch Interaktion lernen, wurde dieses Angebot als willkommene Maßnahme der Integration für geflüchtete Kinder angesehen. Meist als Betreuungsangebot für Kinder von berufstätigen Eltern gedacht, sprach sich die Volksschuldirektorin für den Besuch der Nachmittagsbetreuung insbesondere durch Kinder mit Förderbedarf in Deutsch aus. Durch die gemeinsam verbrachte Zeit werden Freundschaften geknüpft

15 Neben dem simultan bilingualen Erwerb (im Alter von bis zu zwei Jahren) unterscheidet man noch den sukzessiv bilingualen Erwerb (Beginn zwischen zwei und vier Jahren) sowie den kindlichen Zweitsprachenerwerb (Startalter zwischen vier und zehn Jahren) (RoTHwEILER \& RUBERG 2011, pp. 8 f.). 
und die Kinder haben zahlreiche Gelegenheiten die Sprache anzuwenden. $\mathrm{Zu}$ diesem Zweck finanziert die Gemeinde sogar einige Nachmittagsbetreuungsplätze.

Der Schulstandort in der Gemeinde B erhielt im Rahmen des Förderprogramms der Deutschförderklassen und -kurse im Schuljahr 2018/19 erstmals ein größeres staatlich finanziertes Paket für die Deutsch-Sprachförderung, da die benötigte Anzahl an Schüler/inne/n knapp erreicht wurde. Der Förderunterricht zum Erhebungszeitpunkt im Oktober 2018 fand schulklassen-, schulstufen- und schulartübergreifend statt. Die Kinder besuchten grundsätzlich den Regelunterricht, waren auch Teil dieser Klassengemeinschaft, wurden aber für bestimmte Zeiträume aus dem Unterricht herausgenommen, um speziell alle vier Kernkompetenzen (Lesen, Schreiben, Sprechen, Hören) zu üben. Dieses schulorganisatorische Modell der Deutsch-Sprachförderung kann als integratives Modell bezeichnet werden (vgl. von Dewitz \& Massumi 2017, p. 32).

So lernten zum Beispiel ein elfjähriges Mädchen (1. Klasse NMS) und ihr achtjähriger Bruder (3. Klasse VS) aus Afghanistan für die Zeit des Deutschförderkurses gemeinsam. Die Lehrerin gestaltete die Einheit so, dass die Stunde in zwei Blöcke aufgeteilt wurde, um in Kleinstgruppen für je 25 Minuten intensiv mit ihnen zu arbeiten. Auf diese Weise wurde die Konzentrationsspanne der Kinder optimal genutzt. Ihnen stand dazu ein eigener Raum zur Verfügung, der konstant für die Deutschförderaktivitäten genutzt wurde. In diesem Klassenzimmer waren beispielsweise Einrichtungsgegenstände mit Wortkarten beschriftet, die das Vokabellernen unterstützen sollten. Jedes Kind erhielt eigene Aufgabenstellungen, die Lehrerin wechselte stetig zwischen beiden Kindern (Feldnotizen Punz, 24.10.2018).

Diese Art des Unterrichts erforderte von der DaF/DaZ-Förderlehrerin viel Engagement und Kreativität sowie von Seiten der Schulleitung und des Kollegiums hohe Flexibilität und Kooperationsbereitschaft. Für jede Einheit und für jedes Kind mussten Materialien individuell vorbereitet werden; für die Pädagogin war dies ,mit Sicherheit die aufwendigste Stunde der ganzen Woche“ (Interview DAF/DAZ-FöRDERLEHRERIN VS UND NMS GEMEINDE B 2018). Administrativ ist solch ein Fördermodell ebenfalls mit erheblichem Aufwand verbunden, da die Zeiten, in denen die Kinder aus dem Regelunterricht herausgenommen werden, strategisch gut geplant werden müssen. So wurde in der Schule darauf geachtet, dass die Kinder keinesfalls während des Fachs „Bewegung und Sport“ aus dem Klassenverband herausgenommen werden, sondern nach Möglichkeit während der Unterrichtsstunden in den Fächern ,,römischkatholische Religion“" oder „Werken“. Gut abgestimmte Stundentafelplanung zu Beginn des Schuljahres ersetzt allerdings nicht die kontinuierliche enge Zusammenarbeit und regelmäßige Absprachen mit den Klassenlehrer/inne/n. „Inklusion und Kooperation gehören zusammen wie die zwei Seiten einer Medaille' [...]“ (Preuss 2018, p. 53). Dabei ist eine Beziehung, die von gegenseitiger Wertschätzung zwischen Klassenund DaF/DaZ-Förderlehrer/in geprägt ist, enorm wichtig (ebd., p. 56). Wie aus dem folgenden Beispiel hervorgeht, spielt klare Kommunikation innerhalb des pädagogischen Personals eine wesentliche Rolle. 
„Das ist besonders am Anfang sehr schwer. Die Kinder sind den neuen Klassenverband nicht gewöhnt, die neuen Regeln, die neuen Fächer. [...] Und gerade am Anfang die dann rauszunehmen - muss man halt wirklich gut abwägen: Ist das jetzt so wichtig, dass die jetzt 20 neue Vokabeln lernen oder sollten die jetzt vielleicht eine halbe Stunde in der Klasse bleiben und ich helfe ihnen in der Klasse. [...] Also gerade am Anfang ist es nicht leicht. Und da verstehe ich auch jeden Klassenlehrer, der sagt: ,Bitte heute [...] nicht. 'Oder: ,Machen wir es heute so. ' Da ist halt dann auch die Flexibilität einfach gefordert. " (Interview DAF/ DAZ-FöRDERLEHRERIN VS UND NMS GEMEINDE B 2018)

Dem Schulstandort in der Gemeinde B wurden diese Ressourcen 2018 vom Dienstgeber genehmigt, jedoch musste der Direktor von sich aus die Initiative ergreifen, sich selbstständig informieren und darum ansuchen. Trotz dieses Fördermodells griff die Schule weiterhin auf das ehrenamtliche Engagement einiger Senior/inn/en in der Gemeinde zurück. Die Schule hatte sich über die Jahre hinweg ein eigenes DeutschSprachförderprojekt „Senior/inn/en-Lehrer/innen“" aufgebaut. Dadurch, dass im Ort schon seit vielen Jahren Dauergrundversorgungsquartiere bestehen, hatte die Schule kontinuierlich Erfahrung mit der Einschulung außerordentlicher Schüler/innen. Zum Erhebungszeitraum im Oktober 2018 leistete ein Team von circa zehn Pensionist/inn/ en bereits seit vier Jahren wöchentlich 20 Stunden ehrenamtlichen Förderunterricht für außerordentliche Schüler/innen. Eine Lehrerin übernahm die Organisation und Koordination des Projekts. Bei der Planung wurde darauf geachtet, dass die Fördereinheiten zu günstigen Zeiten stattfanden (beispielsweise während des römisch-katholischen Religionsunterrichts). Die Kinder arbeiteten mit den Senior/inn/en in einem eigenen Raum in Kleinstgruppen (ein bis zwei Kinder pro Ehrenamtliche/m/r). Meist bereitete die/der Klassenlehrer/in das Material vor, das in den Stunden mit den Senior/inn/en bearbeitet werden sollte, wobei das spielerische Lernen nicht zu kurz kam. Im Laufe der Zeit wurden die Senior/inn/en so auch zu wichtigen Bezugspersonen im Leben der Kinder (Interview DiREKTOR NMS GEMEINDE B 2018; Interview SENIOR/INN/EN-LEHRER/INNEN GEMEINDE B 2018).

In Gemeinde A konnte die Volksschule sowie die Neue Mittelschule vermehrt seit 2015 Erfahrungen mit der Aufnahme von Kindern und Jugendlichen mit marginalen Deutschkenntnissen sammeln. In den Jahren 2015 und 2016 waren die Pädagog/inn/en sowie die Direktor/inn/en plötzlich vor die Aufgabe gestellt, diese Kinder im Rahmen des Regelunterrichts zusätzlich zu fördern. Diese Herausforderung wurde an beiden Standorten in der Form bewältigt, dass entweder Lehrer/innen in ihren Freistunden die Kinder aus der Klasse holten, um dem Inklusionsanspruch durch die nötige individuelle Förderung gerecht zu werden oder bereits pensionierte Lehrer/innen zusätzlichen Deutschunterricht leisteten. In der Volksschule in Gemeinde A waren Ressourcen für Kinder mit sonderpädagogischem Förderbedarf (SPF) bereits vorhanden, wobei die geflüchteten Kinder auch von diesen Zusatzfördermaßnahmen profitieren konnten.

„Also wir haben eine Klasse mit lernbehinderten und schwerstbehinderten Kindern - wir leben das auch in dem Bereich. Und ich meine, eigentlich kommt 
es sehr viel darauf an, was am Standort gemacht wird. Also man kriegt nicht sehr viel Unterstützung - weder Personal, weder sonst irgendetwas. Wir haben da versucht selber Ressourcen zu schöpfen. Eben Kolleginnen, die schon in Pension sind - zum Teil, für die Sprachförderung und so weiter. " (Interview DiREKTORIN VS GEMEINDE A 2018)

Eine ähnliche Ad-hoc-Lösung schilderte die Schulleiterin der NMS in St. AndräWördern. Sie kritisierte, dass die Bildungsverwaltung angesichts der Entwicklungen 2015 zu wenige Vorkehrungen getroffen hatte und die Schulen mit der Situation alleine gelassen wurden. Damals wurde kein geeignetes Lehrmaterial für den Unterricht von geflüchteten Kindern zur Verfügung gestellt, geschweige denn Personal aufgestockt. An ihrer Schule konnte eine der NMS nahestehende pensionierte Lehrerin für die dringend notwendige zusätzliche Förderung jener Schüler/innen, die zu Beginn kein Wort Deutsch sprachen, gewonnen werden.

Teilweise stellen die sprachlichen Verständigungsschwierigkeiten zwischen Schüler/inne/n, Eltern und Lehrer/inne/n auch erhebliche Hürden abseits des Unterrichtens dar. Die Schulleiterin der NMS St. Andrä-Wördern erinnerte sich an eine Situation, als ein geflüchtetes Mädchen plötzlich weinend vor ihr stand und nicht mehr zu beruhigen war. Da sie sich nicht auf Deutsch ausdrücken konnte und niemand zugegen war, der/die Übersetzungsarbeit leisten hätte können, sah sie sich gezwungen, das Kind von österreichischen Vertrauenspersonen der Familie abholen zu lassen. Solche Momente sind durchaus von Überforderung und vom Gefühl mit der Situation alleine gelassen zu werden geprägt.

Im Rahmen der Elternarbeit ist die Notwendigkeit des Einsatzes von Übersetzer/ inne/n ebenfalls ein wichtiges Thema (vgl. UNHCR ÖsTERREICH 2016, p. 74). Häufig können für terminlich geplante Elterngespräche von den Eltern selbst Dolmetscher/ innen organisiert werden, zum Beispiel im Rahmen der mobilen Flüchtlingsbetreuung für Asylwerber/innen. An der Volksschule in St. Andrä-Wördern fungierte der islamische Religionslehrer als Übersetzer aber auch als Vermittler, was die Schulleiterin besonders schätzte:

„Der islamische Religionslehrer ist ein Tunesier, spricht fließend Türkisch, ausgezeichnet Französisch, [...] und Arabisch und der ist ein sehr, sehr kluger Mann. Ein Akademiker, der uns wirklich in vielen Situationen schon geholfen hat. [...] Er ist ein Mann, hat natürlich in diesem islamischen System einen anderen Stellenwert. Er unterrichtet Religion, ist auch in Familien schon gefahren und hat das Gespräch gesucht. [...] Wir haben eine palästinensische Familie schon seit [...] gut sieben Jahren hier im Haus. Der Vater ist Imam. Da gab's schon immer diese Diskussionen mit Schwimmen gehen, Umkleiden im Turnsaal; diese typischen ,was dürfen Mädchen [Sachen] ' und da war er zum Beispiel federführend daran beteiligt, dass wir diese Barrieren überwunden haben. " (Interview DIREKTORIN VS ST. ANDRÄ-WÖRDERn 2018) 


\section{Exkurs: Muttersprachlicher Unterricht als Anerkennung von sprachlicher Diversität}

Neben den zusätzlichen Maßnahmen zur Verbesserung der Deutschkenntnisse, kann im Rahmen einer umfassenden Sprachförderung muttersprachlicher Unterricht angeboten werden. Expert/inn/en weisen darauf hin, dass sich ausgeprägte Kompetenzen in der Erstsprache positiv auf den Zweit- beziehungsweise Fremdsprachenerwerb auswirken (vgl. RothweILER \& RUBERG 2011, pp. 19 f.). Grundsätzlich besteht in Österreich für Kinder mit einer anderen Erstsprache als Deutsch die Möglichkeit muttersprachlichen Unterricht zu erhalten. Im Schuljahr 2018/19 nahmen nur knapp 14 Prozent aller Schüler/innen der Zielgruppe dieses Bildungsangebot in Anspruch (Bundesministerium FÜR BILdUng, Wissenschaft und Forschung 2019, p. 9). Da viele ländliche Schulstandorte beziehungsweise Schulcluster von nur wenigen Schüler/ inne/n mit nichtdeutscher Muttersprache besucht werden, gestaltet sich die Organisation häufig schwierig und der Unterricht ist in vielen Regionen unter den bestehenden Rahmenbedingungen nicht durchführbar. Ein Blick auf die Liste aller Schulstandorte Österreichs mit muttersprachlichem Unterricht im Schuljahr 2018/19 zeigt, dass beispielsweise Arabisch in Niederösterreich fast ausschließlich in Stadtgemeinden angeboten wurde, Unterricht in Farsi/Dari gab es überhaupt nur an fünf Standorten in Wien (Schule Mehrsprachig 2020). Gelingt es Ressourcen dafür zu beantragen, kann der Unterricht entweder am Nachmittag (Kursunterricht) oder in den Fachunterricht integriert (Teamunterricht) abgehalten werden. Letzterer kann nur dann stattfinden, wenn eine entsprechend hohe Anzahl an Schüler/inne/n Unterricht in ihrer Erstsprache wünschen. In Niederösterreich stellte der Kursunterricht mit gut $70 \%$ im Schuljahr 2018/19 die häufigere Form des muttersprachlichen Unterrichts dar. Wien war das einzige Bundesland, in welchem der muttersprachliche Teamunterricht überwog (BUNDESMINISTERIUM Für BILdUNG, WisSENSCHAFT UND FORSCHUNG 2019).

In den untersuchten Gemeinden im Mostviertel war muttersprachlicher Unterricht kein Thema; wahrscheinlich aufgrund der geringen Anzahl an Schüler/innen, für die dieses Angebot in Frage gekommen wäre. Die Autorin der statistischen Auswertung zum muttersprachlichen Unterricht für das Schuljahr 2018/19 (ebd., pp. 40 f.) nennt einige pragmatische Faktoren, die die Verbreitung des muttersprachlichen Unterrichts bestimmen. Dazu zählen unter anderem: die Anzahl der Personen, welche diese Sprache sprechen und deren räumliche Verteilung, die Verfügbarkeit von qualifizierten Lehrer/ inne/n und die finanziellen Mittel, die dafür bereitgestellt werden. Darüber hinaus sind die Einstellung der Mehrheitsgesellschaft sowie die Förderung von sprachlicher Diversität mitentscheidend. Ebenso spielt die Haltung gegenüber muttersprachlichem Unterricht an den jeweiligen Schulstandorten, in den Familien sowie das Interesse der Schüler/innen selbst eine große Rolle. Schließlich ist festzuhalten, dass ,die pädagogische, bildungspolitische und gesellschaftliche Bedeutung, die mehrsprachiger Bildung im Allgemeinen und dem muttersprachlichen Unterricht im Besonderen beigemessen wird" wesentliche Faktoren sind, die die Entwicklung des Angebots bestimmen (ebd., p. 
40). Dabei ist es wichtig, diese Maßnahmen als Ergänzung und nicht als Ersatz für eine entsprechende Deutschförderung zu sehen.

\subsubsection{Zum Umgang mit den Fluchterfahrungen von Schüler/inne/n}

Die Integration geflüchteter Kinder und Jugendlicher umfasst eine Vielzahl von Aspekten, die über das Erlernen von Deutsch hinausgeht. DASCHNER (2017, pp. 16 ff.) beschreibt mit einigen Schlagwörtern gewisse Charakteristika, die ein differenziertes Bild der/des geflüchteten Schüler/in/s zeichnen:

Es ist davon auszugehen, dass viele Kinder und Jugendliche aufgrund ihrer Erlebnisse vor und während der Flucht traumatisiert sind und an einer posttraumatischen Belastungsstörung leiden. Trauma-Arbeit und der situationsadäquate Umgang mit traumatisierten Kindern und Jugendlichen aus Kriegs- und Krisengebieten in den Schulen stellt ein enorm wichtiges Thema dar, dem oftmals nicht die notwendige und professionelle Aufmerksamkeit geschenkt werden kann. Da enge Bezugspersonen, wie beispielsweise Eltern, oftmals nicht genügend Unterstützung bieten können, da sie selbst traumatisiert sind und keine diesbezügliche Ausbildung aufweisen, weist DASCHNER auf den dringenden Bedarf an psychologischen Unterstützungsangeboten für Pädagog/inn/ en an den betroffenen Schulen hin (ebd., p. 17). Der Direktor der NMS in Gemeinde A beklagte beispielsweise, dass präventiv keine Ressourcen zur Verfügung gestellt wurden. Schulpsychologische Unterstützung würde erst im Anlassfall geleistet. Das UNHCR-Handbuch für Pädagog/inn/en zum Thema Flucht und Trauma in der Schule (UNHCR ÖSTERREICH 2016) stellt wichtige Hintergrundinformation für Pädagog/inn/ en bereit. Beispielsweise gibt das Handbuch Tipps für Erste-Hilfe-Maßnahmen bei einer möglichen Retraumatisierung durch Trigger im Schulalltag und stellt Routinen und Verhaltensweisen vor, die Kindern dazu verhelfen, die Schule als sicheren Ort wahrzunehmen. Darüber hinaus liefert es Anregungen für die traumapädagogische Gesprächsführung sowie die Elternarbeit.

Die Kinder sind sprach-los, im doppelten Sinn. Einerseits verstehen sie anfangs die Sprache nicht und können sich nicht ausreichend artikulieren, andererseits können sie das Erlebte oft nicht in geeignete Worte fassen. UNHCR ÖSTERREICH (2016, p. 43) weist darauf hin, dass insbesondere Kinder, die zur Zeit der Traumatisierung sehr jung waren, möglicherweise in ihrer Erstsprache noch nicht den nötigen Wortschatz erworben hatten, um ihre Gefühle ausdrücken zu können. Dies hat Auswirkungen auf das Lernen weiterer Fremd- oder Zweitsprachen. Oftmals zeichnen sich in diesem Fall anfangs kaum Fortschritte beim Spracherwerb ab.

DASCHNER (2017, p. 17) erläutert, dass Kindern aufgrund ihrer Sozialisation in einem anderen Land anfangs alles fremd ist - nicht nur die Sprache, auch der (schulische) Alltag in Österreich ${ }^{16}$. Die Schüler/innen kommen häufig aus autoritär geführten Staaten

$16 \quad$ Auf den Aspekt der kulturellen Unterschiede wird hier nicht näher eingegangen, da er nicht im Fokus der Erhebung stand. In den Interviews wurden diesbezüglich einige Konfliktfelder angeschnitten, die aber nicht weiter vertieft wurden: das Tragen des Kopftuches in der Schule 
mit hierarchischen Familienstrukturen, die durch väterliche Dominanz geprägt sind. Hier in Österreich erfahren sie eine andere schulische Sozialisation, in deren Rahmen selbständiges Lernen, Eigenverantwortlichkeit, Mitbestimmung sowie die Artikulation der eigenen Meinung erwartet werden.

Geflüchtete Kinder und Jugendliche sind aber auch eine extrem heterogene (DASCHNER 2017, p. 16) Gruppe. Nicht nur was ihren formalen Bildungsstand anbelangt, bringen Kinder und Jugendliche jeden Alters unterschiedliche Voraussetzungen mit. Die Entwicklung der Kinder als Teil einer Klassengemeinschaft ist sehr individuell. Abgesehen von kognitiven Begabungen können sich Schüler/innen auch schneller oder langsamer an neue Umgebungen, Strukturen und Regeln gewöhnen. Manche Kinder schließen sehr schnell Freundschaften, andere nicht. Im Umgang mit traumatischen Erfahrungen besitzt jedes Kind eine persönliche Resilienz. Manche kommen mit der Verarbeitung schlimmer Erlebnisse besser zurecht als andere (UNHCR ÖsterReICH 2016, p. 77). Pädagog/inn/en haben die Erfahrung gemacht, dass sich Kinder unabhängig von ihrer Fluchterfahrung sehr unterschiedlich entwickeln. Nicht nur geflüchtete Kinder haben individuelle (Förder-)Bedürfnisse, auch österreichische Schüler/innen mit deutscher Erstsprache sind nicht als homogene Gruppe zu betrachten. Generalisierungen in jegliche Richtung sind eher hinderlich, um das Phänomen der Integration geflüchteter Schüler/innen in der Pflichtschule zu erfassen. Daher scheint ein One-size-fits-all- Modell für die Beschulung und Integration dieser Schüler/innen wenig erfolgversprechend. Vielmehr sind interkulturelle Fähigkeiten der Lehrer/innen und Rahmenbedingungen gefragt, die es ihnen erlauben, ihren Unterricht zu individualisieren (DASCHNER 2017, p. 16).

„Inklusion birgt den zutiefst originär pädagogischen Anspruch, alle Kinder und Jugendlichen in der Schule gerecht zu bilden, sie optimal zu fördern und jeder einzelnen Schülerin, jedem einzelnen Schüler unabhängig von ihrer/seiner Behinderung, der sozialen, ethnischen, kulturellen, religiösen, gesellschaftlichen oder begabungsbedingten Herkunft maximale Teilhabe zu ermöglichen." (Preuss 2018, p. 11)

Aus dieser Textstelle geht ein weites Verständnis von Inklusion ${ }^{17}$ hervor - eines, welches verschiedene von Diskriminierung betroffene Gruppen, zum Beispiel eben auch Kinder und Jugendliche mit Fluchtbiografie, miteinschließt. Bewerkstelligt werden

(insbesondere beim Turnunterricht), der Schwimmunterricht für Mädchen, das Umkleiden für den koedukativen Sportunterricht in der Volksschule (insbesondere das gemeinsame Umkleiden) und das (unentschuldigte) Fernbleiben vom Unterricht anlässlich der muslimischen Feiertage. LUCIAK \& Binder (2010, pp. 68 ff.) liefern praxisnahe Hinweise, wie im Schulalltag damit konstruktiv umgegangen werden kann.

17 Zur Verwendung der Begriffe „Integration“ und „Inklusion“ vgl. Preuss (2018, pp. 17 ff.). Das originäre Konzept der Inklusion beschreibt das gemeinsame Lernen von Kindern mit und ohne sonderpädagogischem Förderbedarf. Inzwischen fand eine Verschmelzung des Begriffs mit dem Diskurs über Heterogenität, Migration und Integration statt. Das Konzept „Inklusion“ wird daher vor allem im Zusammenhang mit Bildungsthematiken häufig synonym mit dem Begriff „Integration“" verwendet. 
kann dies nur durch die Bereitstellung von Ressourcen für die individuelle Förderung (Preuss 2018, pp. 17 f.). Trotz der knappen und teilweise nicht existenten Mittel für diese individuelle Förderung, versuchen die Schulen in den beiden untersuchten Regionen eigene Ressourcen zu organisieren. Begründet wird dieses Engagement auch mit einem ,Lehrer/innen-Ethos“. Die Volksschuldirektorin in Gemeinde A drückte es folgendermaßen aus: „Es hat sich jeder bemüht um das Kind. Man sieht das Kind und damit ist auch das Bemühen da.“

Die Figur des geflüchteten Kindes spielt im Hinblick auf den Diskurs um „,deserving refugees"18 eine wesentliche Rolle. Auch dadurch, dass Kinder unverschuldet in diese prekäre Situation gelangt sind, wird ihnen Empathie zuteil. „Die Eltern haben sich zur Flucht entschieden, die Kinder haben sich nie entschieden. Die Kinder sind immer Mitfahrer.“ (Interview DirekTor NMS GemeInde B 2018). Eine ähnliche Perspektive wurde im Interview mit der Schulleiterin der NMS in St. Andrä-Wördern ausgedrückt, die besorgt auf die Zeit während der Flucht als ,,verlorene Zeit“ für die Bildungskarriere der Kinder blickt. In der alltäglichen Praxis von Schulen sowie im Unterricht ist es allerdings auch wichtig, Kinder und Jugendliche mit Fluchterfahrung nicht ständig und ausschließlich als hilfsbedürftig und bemitleidenswert zu betrachten.

„,[Ich] muss eher aufpassen, dass nicht so ein hysterischer Helferwahn passiert, ja? Es war wirklich so. Als wir die ersten Kinder bekamen - ich kann mich noch gut erinnern, ein kleines tschetschenisches Mädchen, - da sind am nächsten Tag fünf Schultaschen da gewesen, sechs Federpennale, ich weiß nicht, Bücher, Materialien, Spielzeug. [...] Wir haben Eltern mit einem sehr hohen Sozialniveau und manchmal hab'ich fast Angst, dass da die Kinder fast zu so einem Klassenmaskottchen werden. Das versuche ich auch zu verhindern, deswegen sind mir die Verweilzeiten am Nachmittag so wichtig. [...] Weil Integration heißt ein Teil einer Gruppe zu sein und nicht, der Arme', dem es zu helfen gilt rund um die Uhr. Das ist ein falscher Ansatz und die Gefahr besteht, wenn man's übertreibt, ja?"“ (Interview DireKTORIN VS ST. ANDRÄ-WÖRDERn 2018)

Eine Reduktion des Problemdiskurses im Hinblick auf speziellen sprachlichen Förderbedarf bei Kindern und Jugendlichen mit Fluchterfahrung greift ebenfalls zu kurz. Sprachwissenschafter/innen und Pädagog/inn/en weisen darauf hin, dass die generelle Einstellung der Eltern zu Bildung maßgeblich darauf Einfluss nimmt, wie sich Kinder

18 Zum Diskurs des „deserving refugee“ vgl. Holmes \& CASTAÑEDA (2016) sowie Ravn et al. (2020). RAVN et al. (2020, pp. 136 f.) beschreiben drei Dimensionen von „deservingness“ im Kontext von Flucht: Die erste Dimension orientiert sich an der Genfer Flüchtlingskonvention und der darin festgeschriebenen Definition von Geflüchteten. Die zweite Dimension beschreibt ,deservingness" vor dem Hintergrund eines meritokratischen Prinzips. Jenen (Flucht-)Migrant/inn/en, die den Wohlfahrtsstaat nicht belasten und die produktive Arbeitnehmer/innen beziehungsweise Unternehmer/innen sind, sollte ein Aufenthaltsrecht gewährt werden. Schließlich beschreibt die dritte Dimension ,deservingness“ in Verbindung mit Vulnerabilität. Dabei spielen Fremdzuschreibungen eine wesentliche Rolle. Gemeinhin werden Frauen und Kinder als am vulnerabelsten angesehen. Dieses Konzept steht in enger Verbindung mit Vorstellungen des Opferseins und zumindest stark eingeschränkter Agency. 
in der Schule entwickelt. Dabei spielt auch die Pflege der Erstsprache in den Familien eine wichtige Rolle (RothweILER \& RUBERG 2011, pp. 19 f.).

„Ich werd' das immer wieder von Freunden gefragt: , Wie viele Ausländer hast du an deiner Schule?' Das ist absurd, weil ich hab ' [...] österreichische Kinder mit massiven Sprachdefiziten, die sich in Zwei-Wort-Sätzen unterhalten, wenn ich sie aufnehme. Und ich hab' dieses berühmte syrische Mädchen, das mir nach zwei Jahren hier im System fehlerlose Aufsätze, zweiseitige, geschrieben hat in höchster Sprachqualität. [...] Es ist hochgradig milieuabhängig und abhängig von der Förderung im Elternhaus. " (Interview DiREKTORIN VS ST. ANDRÄ-WöRDERN 2018)

Das zeigt, dass es nicht zu rechtfertigen ist, geringe Sprachkenntnisse nur an der Herkunft der Schüler/innen festzumachen. Generalisierungen, welche Kinder mit nichtdeutscher Erstsprache oder mit Fluchterfahrung von vornherein als Problemgruppe gelten, sind demnach nicht zulässig. Stattdessen sollte man sich vermehrt mit der Frage auseinandersetzen, wie jene Kinder, die auf sprachlicher Ebene Unterstützungsbedarf haben, am besten gefördert werden können. Die Pflege der Muttersprache spielt hier ebenfalls eine wichtige Rolle, da der Erwerb von Kenntnissen in anderen Sprachen auch davon abhängt, auf welchem Niveau die Erstsprache beherrscht wird (SCHMIDINGER 2010, p. 40).

Aus der Forschung zu den Bildungsaspirationen von Migrant/inn/en für ihre Kinder ist bekannt, dass diese häufig im Vergleich zur Bevölkerung des Aufnahmelandes überdurchschnittlich hoch sind (vgl. zum Beispiel BECKER 2010; LACHMAYR, LEITGÖB \& BACHER 2011). Auf Geflüchtete scheint dies ebenso zuzutreffen, wie die Ergebnisse einer qualitativen Studie zu den Einstellungen, Erwartungen und Ressourcen weiblicher Flüchtlinge von BIFfL, KILIC \& ZENTNER (2019, p. 76) vermuten lassen. Teilweise konnten auch die Perspektiven der jeweiligen Ehemänner erhoben werden. Mitunter hatten die Eltern selbst in ihrem Herkunftsland nicht die Möglichkeit die Schule zu besuchen oder einen Bildungsabschluss zu erlangen. Ihnen ist die Bildung für ihre Kinder in Österreich häufig sehr wichtig. Allerdings können sie diese - nicht zuletzt wegen der unzureichenden Deutschkenntnisse - kaum unterstützen (vgl. DASCHNER 2017, p. 16). Die Direktorin der Volksschule in St. Andrä-Wördern berichtete beispielsweise von einer afghanischen Familie, bei der es sehr schwer war, schulische Inhalte zu vermitteln. Beide Eltern waren Analphabeten, weshalb sich die Lehrer/innen verstärkt auf die Kommunikation mit den Kindern verlassen mussten. Es wird sich in den nächsten Jahren noch zeigen, ob es geflüchteten Schüler/inne/n gelingen wird im österreichischen Schulsystem zu bestehen und ihre Bildungsaspirationen, beziehungsweise die ihrer Eltern, erfolgreich umzusetzen. Weitere empirische Forschungen in diesem Bereich sind daher in jedem Fall notwendig, um diesen Prozess auch entsprechend wissenschaftlich zu erfassen. 


\subsection{Fazit}

$\mathrm{Zu}$ Beginn des Kapitels wurde ein Analysemodell vorgestellt, das den Bereich der Schulbildung auf drei Ebenen erfasst: Das nationale Bildungssystem bildet mit seinen Strukturen die Makroebene. Auf der Mesoebene befindet sich die Einzelschule, also ein Schulstandort, der mit einem gewissen Leitbild ausgestattet ist und spezifische Werte vertritt. Auf der Mikroebene, also der Ebene der individuellen Akteur/inn/e/n, stehen persönliche Interaktionen zwischen Lehrer/inne/n, Schüler/inne/n und Eltern im Vordergrund (PILZ 2016, pp. 48 ff.).

Im Rahmen der Feldforschung wurden hauptsächlich Direktor/inn/en zu den Herausforderungen und Erfolgen bei der Integration geflüchteter Schüler/innen befragt. Dabei wurde das Spannungsfeld zwischen den Strukturen des Bildungssystems (Makroebene) und den zu bewältigenden Aufgaben bei der Integration dieser Kinder am Schulstandort (Mesoebene) thematisiert. Es konnte gezeigt werden, welche Gestaltungsspielräume Schulstandorte innerhalb der strukturellen Vorgaben auf der Makroebene besitzen und wie diese genützt werden. Die Interaktionen zwischen Lehrer/inne/n und Schüler/inne/n (Mikroebene) sowie die eigentliche Vermittlung von Inhalten standen nicht im Fokus der Erhebung.

Dass die Integration der geflüchteten Schüler/innen in den beiden Regionen so gut funktioniert, hängt maßgeblich damit zusammen, dass an allen Schulen kreativ interne Wege für die zusätzliche Förderung, vor allem in der deutschen Sprache, gefunden wurden. Dabei kamen integrative Modelle (vgl. von Dewitz \& Massumi 2017, p. 32) zum Einsatz, das heißt, die Kinder wurden zu gewissen Zeiten aus dem Regelunterricht herausgeholt, um spezielle Deutschförderung zu erhalten. Diese Maßnahmen können als Ad-hoc-Lösungen bezeichnet werden, da vom Dienstgeber anfangs keine Mittel (Personal, bezahlte Unterrichtsstunden, Lehrmaterial) zur Verfügung gestellt wurden. Die untersuchten ländlichen Regionen hatten jeweils nur wenige geflüchtete Kinder und Jugendliche aufgenommen. Diese Tatsache wurde zwar einerseits als positiv bei der Aufgabenbewältigung angesehen, andererseits reichte die Anzahl außerordentlicher Schüler/innen an den Schulen meist nicht aus, um eine Genehmigung für bezahlte Unterrichtsstunden zur zusätzlichen Deutschförderung zu erhalten.

So viel eigenes Engagement in dieser Sache ist keine Selbstverständlichkeit und zeigt, dass die Schulstandorte offen für den Umgang mit einer sprachlich und ethnisch diversen Schüler/innen/gruppen sind. Dabei wird intern von der Schulleitung sowie vom Kollegium weitgehend an einem Strang gezogen, denn individualisierter beziehungsweise inklusiver Unterricht verlangt Planung und Kooperation von den Pädagog/ inn/en. Das erfordert Absprachen und Koordination, beispielsweise wenn ein Kind vorübergehend aus der Klasse genommen werden soll (vgl. PReuss 2018, pp. 53 ff.).

Anders als bei Pilz (2016, pp. 41 ff.) beschrieben, wurden geflüchtete Kinder und Jugendliche trotz der Herausforderungen, die ihre Aufnahme für die Schulstandorte 
bedeutete, nicht vordergründig als Problem angesehen (vgl. Abschnitt 5.1). Die Direktor/ inn/en äußerten sich vielmehr kritisch über das starre und bürokratische Schulsystem, das an den Standorten generell nur wenig Flexibilität zulässt. Andererseits zeigte sich auch, dass Einzelschulen und Schulleitungen willig und fähig sind, unkonventionelle Lösungen für plötzliche Herausforderungen zu finden.

Schulleiter/innen sind Expert/inn/en für ihren Schulstandort. Sie wissen meist am besten, welche Ressourcen benötigt und wie diese in geeigneter Weise eingesetzt werden können. Für manche Schulen mag ein teilintegratives oder paralleles Modell sinnvoll sein, für andere Standorte eher ein integratives. Die Wirksamkeit der verschiedenen schulorganisatorischen Modelle dürfte auch mit der Anzahl und dem Alter der Schüler/ innen zusammenhängen. Jedenfalls wünschen sich Schulleitungen mehr Unterstützung bei der Bewältigung der Integration durch die Bereitstellung von Ressourcen, die sie selbständig verwalten können. Selbst wenn nur wenige geflüchtete Schüler/innen einen Schulstandort besuchen und möglicherweise während des laufenden Schuljahres eingeschult werden müssen oder die Schule auch wieder verlassen, braucht es Mittel und Wege, die die Förderung dieser Kinder und Jugendlichen unterstützen. Ausschließlich im Rahmen des Regelunterrichts ist diese komplexe Aufgabe kaum zu bewältigen. 